\title{
GRZYBY MAKROSKOPIJNE REZERWATU PRZYRODY „TORFOWISKO TOPORZYK” (NW POLSKA)
}

\author{
MAŁGORZATA STASIŃSKA, ZOFIA SOTEK \\ Katedra Botaniki i Ochrony Przyrody, Wydział Biologii, \\ Uniwersytet Szczeciński, \\ ul. Felczaka 3c, 71-412 Szczecin
}

\begin{abstract}
The paper presents results of mycological research carried out in the "Torfowisko Toporzyk" nature reserve (NW Poland), in the years 2004-2009. As a result of the studies, 216 fungal species were identified, 17 Ascomycota and 199 Basidiomycota. Twenty of the recorded species are included on the Polish red list of macrofungi, e.g. Cortinarius violaceus, Lactarius lacunarum, Mycena megaspora and Suillus flavidus. Among the plant associations, Vaccinio uliginosi-Pinetum (87 species) and Vaccinio uliginosi-Betuletum pubescentis (77), turned out to be the richest in fungi, whereas the fewest taxa were found in Rhynchosporetum albae (8).
\end{abstract}

Keywords: macromycetes, threatened species, raised peat-bog, Pomerania, Poland

\section{WSTĘP}

Rezerwat przyrody „Torfowisko Toporzyk”, znajdujący się w otulinie Drawskiego Parku Krajobrazowego, został utworzony 12 listopada 1996 r. (Zarządzenie... 1996) w celu zachowania, ze względów naukowych i dydaktycznych, torfowiska $\mathrm{z}$ reliktowymi zbiorowiskami roślinnymi. Torfowisko w przeszłości zostało w znacznym stopniu wyeksploatowane i obecnie zachodzi na nim naturalna sukcesja w kierunku odnowienia zbiorowisk torfotwórczych. Głównym przedmiotem ochrony jest regenerująca się roślinność mszarna w dołach potorfowych oraz bory i brzeziny bagienne. O dużych wartościach przyrodniczych tego obiektu donosili Jasnowska i Jasnowski (1983). Prowadzone były tam badania przede wszystkim o charakterze florystycznym i fitosocjologicznym, które były prezentowane w pracach dotyczących wyłącznie tego obiektu (Zabawski, Matuła 1975; Brzeg i in. 1992, 1995). Badania nad florą rezerwatu realizowano także w ramach fitogeograficznych studiów, dotyczących roślin naczyniowych torfowisk Pomorza (Sotek 2010). W ramach prac związanych z przygotowaniem planu ochrony rezerwatu przeprowadzono szczegółową inwentaryzację geobotaniczną i faunistyczną, a także zdefiniowano zagrożenia i zaplanowano działania ochronne (Jasnowska i in. 2003-2004). Ze względu na rzadką i cenną szatę roślinną rezerwatu oraz zachodzące w niej zmiany rozpoczęto obserwacje 
mykologiczne, które, obok badań faunistycznych, są niezbędne do poznania pełnej wartości tego chronionego obiektu.

Celem badań było określenie bogactwa i różnorodności gatunkowej grzybów makroskopijnych rezerwatu przyrody „Torfowisko Toporzyk”. Ich wyniki były już częściowo publikowane i dotyczyły zróżnicowania macromycetes w zespołach roślinnych Erico-Sphagnetum magellanici (Stasińska, Sotek 2010; Stasińska 2011) i Caricetum lasiocarpae (Stasińska 2011). Niniejsza praca zawiera wykaz wszystkich stwierdzonych gatunków w badanych zbiorowiskach torfowiskowych mszarnych i leśnych oraz we fragmentarycznie wykształcających się zbiorowiskach lasów na siedliskach mineralnych rezerwatu.

Praca była częściowo finansowana ze środków na naukę w latach 2007-2009 w ramach projektu badawczego MNiSW nr N N305 261733.

\section{TEREN BADAŃ}

Rezerwat „Torfowisko Toporzyk”, o powierzchni 43,07 ha, znajduje się po wschodniej stronie wsi Dobino, około 1,5 km na północ od miejscowości Toporzyk, w gminie Połczyn Zdrój (ryc. 1). Prawie ze wszystkich stron otoczony jest polami uprawnymi. Torfowisko wykształciło się na obszarze wododziałowym dorzeczy Wogry i Rakonu. Jego środek przecina, z północy na południe, pasmo pagórków mineralnych o wysokości około $150 \mathrm{~m}$ n.p.m. Wśród gleb dominują gleby torfowe i brunatne wyługowane, występują także gleby murszowo-torfowe i opadowo-glejowe, głównie na obrzeżach torfowiska, zajmując niewielkie powierzchnie. W przeszłości wydobywano tam torf i w związku z tym teren ten podlegał odwodnieniu poprzez system rowów melioracyjnych. Obecnie niektóre wyrobiska potorfowe stanowią dość duże zbiorniki z otwartym lustrem wody, natomiast większość rowów jest zarośnięta i tylko w szerszych stagnuje woda. Obrzeża rezerwatu ulegają eutrofizacji w wyniku spływów powierzchniowych z pól uprawnych oraz odprowadzania wody z miejscowości Dobino rowem melioracyjnym łączącym się z rowem opaskowym torfowiska. Ekosystem ten, mimo znacznych przekształceń, nadal pozostaje torfowiskiem żywym, na którym zachodzą procesy regeneracyjne o różnym stopniu zaawansowania.

Obszar, na którym znajduje się rezerwat, podlega większym wpływom klimatu atlantyckiego niż kontynentalnego. Opady roczne, które są jedynym źródłem zasilania torfowiska wysokiego w wodę, w tym rejonie kształtują się na poziomie $725 \mathrm{~mm}$, a średnia roczna temperatura powietrza wynosi $7,5^{\circ} \mathrm{C}$ (Koźmiński i in. 2007).

W roślinności torfowiskowej rezerwatu dominującą rolę pełnią mszary. Występują one głównie w zachodniej części obiektu i wykazują znaczne zróżnicowanie, spowodowane prowadzoną w przeszłości eksploatacją torfu. Wyodrębniają się tam mszary wysokie i przejściowe. Do najcenniejszych mszarów 


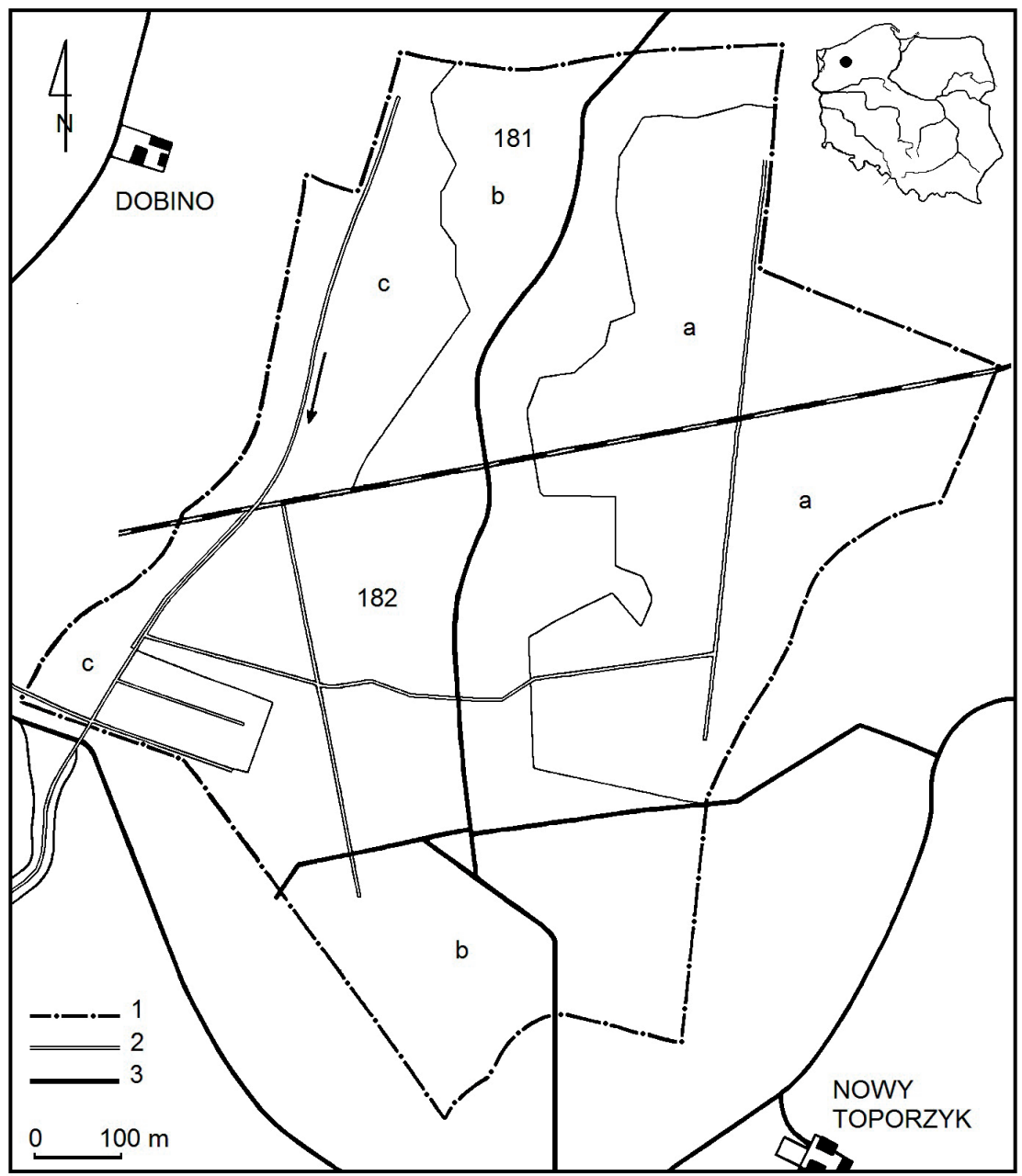

Ryc. 1. Lokalizacja i plan rezerwatu przyrody „Torfowisko Toporzyk” 1 - granica rezerwatu, 2 - rowy melioracyjne, 3 - drogi

Fig. 1. Locality and map of the "Torfowisko Toporzyk" nature reserve 1 - border of reserve, 2 - drainage ditches, 3 - roads

wysokich należy Erico-Sphagnetum magellanici, który osiąga w tym rejonie południową granicę swego zasięgu. Zbiorowisko to ma charakter atlantycki, występuje na kopule torfowiska. Jednym z głównych jego komponentów jest Erica tetralix. Gatunek ten w Polsce jest zagrożony i znajduje się zarówno na regionalnych czerwonych listach roślin (m.in. Żukowski, Jackowiak 1995; Markowski, Buliński 2004), jak i na liście krajowej (Kaźmierczakowa i in. 2016), w której ujmowany jest w kategorii narażony (VU). Przenikające na ten obszar wpływy klimatu kontynentalnego znajdują odzwierciedlenie w roślinności, 
czego przykładem jest mszar wysoki Ledo-Sphagnetum magellanici, stanowiący fazę przejściową od otwartych torfowisk do borów bagiennych. Miejscami odznacza się on zwartym występowaniem Ledum palustre, gatunku o zasięgu cyrkum-borealnym (Zając M., Zając A. 2006). W niektórych starych dołach potorfowych wykształcił się mszar wysoki Sphagnetum magellanici, mający strukturę dolinkowo-kępkową. W dolinkach rosną m.in.: Carex rostrata, Eriophorum angustifolium i Sphagnum fallax, a kępki tworzą m.in.: Andromeda polifolia, Eriophorum vaginatum, Oxycoccus palustris, Sphagnum magellanicum i Sph. rubellum. Mszary przejściowe występują głównie w dołach potorfowych we wczesnych fazach regeneracyjnych roślinności torfowiskowej. Niewielkie powierzchnie porastają: mszar przygiełkowy Rhynchosporetum albae i mszar turzycy nitkowatej Caricetum lasiocarpae, natomiast miejscami rozległe płaty tworzy zbiorowisko, w którym występuje łanowo Carex rostrata. Duży udział w strukturze mszarów przejściowych rezerwatu ma zespół Eriophoro angustifolii-Sphagnetum recurvi.

Znaczne powierzchnie rezerwatu zajmują bory i lasy bagienne (Brzeg i in. 1995). Bór bagienny Vaccinio uliginosi-Pinetum porasta bardziej przesuszone powierzchnie torfowiska i groble. W drzewostanie dominuje Pinus sylvestris, a wśród pozostałych gatunków zaznacza się udział Ledum palustre i Vaccinium uliginosum. Rozległe płaty brzeziny bagiennej Vaccinio uliginosi-Betuletum pubescentis wykształciły się głównie w zachodniej części rezerwatu. Ich drzewostan buduje przede wszystkim Betula pubescens, z domieszką $B$. pendula i $P$. sylvestris. W warstwie zielnej miejscami dość licznie występuje gatunek regionalnie charakterystyczny dla tego zespołu - Lycopodium annotinum. $\mathrm{Na}$ zachodnich skrajach rezerwatu, w sąsiedztwie rowu opaskowego, w miejscach zeutrofizowanych, rozwinęły się zbiorowiska olsowe Sphagno squarrosiAlnetum i zarośla łozowe Salicetum pentandro-cinereae. Łozowiska występują także w innych częściach rezerwatu, w obniżeniach terenu, w miejscach bardziej uwilgotnionych. Wzniesienia mineralne porastają lasy zbliżone do kwaśnej buczyny niżowej Luzulo pilosae-Fagetum i żyznej buczyny niżowej Galio odorati-Fagetum (Jasnowska i in. 2003-2004).

\section{METODY BADAŃ}

Badania mykologiczne w rezerwacie „Torfowisko Toporzyk” prowadzono w latach 2004-2009, głównie w zbiorowiskach torfowiskowych. Do obserwacji wyznaczono stałe powierzchnie badawcze w płatach zespołów: Caricetum lasiocarpae, Eriophoro angustifolii-Sphagnetum recurvi, Erico-Sphagnetum medii, Rhynchosporetum albae, Vaccinio uliginosi-Betuletum pubescentis i Vaccinio uliginosi-Pinetum. Z kolei w zbiorowiskach: Caricetum rostratae, LedoSphagnetum magellanici, Sphagnetum magellanici oraz we fragmentarycznie 
wykształconych Luzulo pilosae-Fagetum i Galio odorati-Fagetum zastosowano metodę marszrutową. Obserwacje prowadzono systematycznie od kwietnia do listopada.

Nomenklaturę grzybów przyjęto za MycoBank (Robert i in. 2005) i Index Fungorum (2017). Nazwy roślin naczyniowych podano za Mirkiem i in. (2002), a nazwy mchów za Ochyrą i in. (2003). Nazewnictwo zbiorowisk roślinnych przyjęto za Matuszkiewiczem (2001) i Herbichową (2004).

Wyniki badań przedstawiono w postaci listy gatunków w układzie alfabetycznym, oddzielnie dla Ascomycota i Basidiomycota; dla każdego gatunku podano: typ podłoża, na którym występował, zbiorowisko roślinne i oddział leśny, w którym został stwierdzony oraz okres występowania (miesiące i lata). Kategorie zagrożenia grzybów podano według Wojewody i Ławrynowicz (2006), a status prawny według Rozporządzenia... (2014). Materiały zielnikowe złożono w zielniku Katedry Botaniki i Ochrony Przyrody Uniwersytetu Szczecińskiego (SZUB-F).

\section{WYNIKI}

W wyniku przeprowadzonych badań w rezerwacie stwierdzono 216 gatunków grzybów makroskopijnych, w tym 17 Ascomycota i 199 Basidiomycota. Wśród odnotowanych taksonów 20 ujętych jest na czerwonej liście grzybów wielkoowocnikowych w Polsce (Wojewoda, Ławrynowicz 2006), w różnych kategoriach zagrożeń. Do kategorii E (wymierających) należą: Cortinarius fulvescens, Galerina jaapii, Lactarius lacunarum i Suillus flavidus, do V (narażonych) - pięć gatunków, m.in. Cortinarius violaceus, Leccinum niveum i Mycena megaspora, a do R (rzadkich) - 11, m.in. Galerina sphagnorum, Phaeomarasmius erinaceus i Pseudomerulius aureus. Trzy z gatunków zagrożonych objęte są ochroną częściową - Inonotus obliquus, Pseudoboletus parasiticus i Suillus flavidus. Większość z odnotowanych gatunków grzybów zagrożonych stwierdzona została w zbiorowiskach torfowiskowych.

Wśród zespołów roślinnych największym bogactwem grzybów odznaczały się zbiorowiska torfowiskowe leśne Vaccinio uliginosi-Pinetum (87 gatunków) i Vaccinio uliginosi-Betuletum pubescentis (77). Znaczną różnorodność grzybów wykazywały również zbiorowiska leśne wykształcające się fragmentarycznie na podłożu mineralnym - Luzulo pilosae-Fagetum (42 gatunki) i Galio odorati-Fagetum (47). Najmniej taksonów stwierdzono w mszarach przejściowych Rhynchosporetum albae (8) i zbiorowisku z Carex rostrata (9). 


\section{WYKAZ GATUNKÓW GRZYBÓW}

Przyjęte skróty: Cala - Caricetum lasiocarpae, Caro - zbior. z Carex rostrata, EaSp - Eriophoro angustifolii-Sphagnetum recurvi, ErSp - Erico-Sphagnetum medii, GF - Galio odorati-Fagetum, LeSm - Ledo-Sphagnetum magellanici, LF - Luzulo pilosae-Fagetum; Rhal-Rhynchosporetum albae, Spma - Sphagnetum magellanici, VuBe - Vaccinio uliginosi-Betuletum pubescentis; VuPn - Vaccinio uliginosi-Pinetum; oddz. - oddział leśny; IV-IX 2009 - okres występowania; $\mathrm{Ch}$ - gatunki objęte ochroną częściową; kategorie zagrożenia: E - wymierające, V - narażone, $\mathrm{R}$ - rzadkie.

\section{Ascomycota}

Ascocoryne sarcoides (Jacq.) J.W. Groves \& D.E. Wilson - na murszejących kłodach Betula; VuBe; oddz. 181c; X-XI 2007-2009.

Bertia moriformis (Tode) De Not. - na gałązkach; GF, LF; oddz. 181b, 182b; VIII-IX 2004-2006. Bisporella citrina (Batsch) Korf \& S.E. Carp. - na drewnie; GF, LF; oddz. 181b, 182b; VII-IX 2004-2005, 2008.

Colpoma quercinum (Pers.) Wallr. - na gałązkach Quercus; GF, LF; oddz. 181b, 182b; V-VI 2004-2005, 2007.

Dasyscyphus virgineus (Batsch) Gray [=Lachnum virgineum (Batsch) P. Karst.] - na martwym drewnie; GF, LF; oddz. 181b, 182b; IV-V 2004-2005, 2007.

Dialonectria episphaeria (Tode) Cooke [=Nectria epishaeria (Tode) Fr.] - na Diatrype stigma; VuBe; oddz. 181c; IV-XI 2007-2009.

Diatrype disciformis (Hoffm.) Fr. - na gałęziach Fagus; GF, LF; oddz. 181b, 182b; V-XI 20042009.

D. stigma (Hoffm.) Fr. - na martwych gałązkach i gałęziach Betula; VuBe; oddz. 181b, c; IV-XI 2004-2005, 2007-2009.

Diatrypella verrucaeformis (Ehrh.) Nitschke - na martwych gałązkach i gałęziach Betula; GF, LF, VuBe; oddz. 181a, b, c, 182a, b; IV-XI 2004-2009.

Hymenoscyphus fructigenus (Bull.) Gray - na owocach Quercus; GF, LF; oddz. 181b, 182b; V-VI 2004-2005.

Hypoxylon fragiforme (Pers.) J. Kickx f. - na gałęziach Fagus; GF, LF; oddz. 181b, 182b; V-X 2004-2009.

Kretzschmaria deusta (Hoffm.) P.M.D. Martin [=Ustulina deusta (Hoffm.) Maire] - na pniakach Fagus; GF, LF; oddz. 181b, 182b; V-VI 2004-2006, 2009.

Mollisia cinerea (Batsch) P. Karst. - na martwym drewnie Betula; VuBe; oddz. 181c; VII-IX 2008-2009.

Nectria cinnabarina (Tode) Fr. - na martwych gałązkach Betula i Fagus; GF, LF, VuBe; oddz. 181b, c, 182b; IX-XI 2004-2009.

Peziza vesiculosa Bull. - na ziemi; GF; oddz. 181b; IX 2005.

Scutellinia scutellata (L.) Lambotte - na murszejących gałęziach Betula; VuBe; oddz. 181c; VIIIIX 2007-2009.

Xylaria hypoxylon (L.) Grev. - na martwym drewnie, na pniakach; $G F, L F, V u B e$; oddz. 181b, c, 182b; IV-XI 2004-2005, 2007-2009.

\section{Basidiomycota}

Amanita citrina (Schaeff.) Pers. - na ziemi i torfie; VuBe; oddz. 181b, c; IX 2006-2007.

A. fulva Pers. - na torfie, wśród Sphagnum; GF, ErSp, VuBe, VuPn; oddz. 181a, b, c, 182a, b; VII-X 2004-2005, 2007-2009. 
A. muscaria (L.) Lam. - na ziemi, pod Betula; oddz. 181c; VII-IX 2004-2006.

A. pantherina (DC.) Krombh. - na ziemi; GF; oddz. 181b; IX 2005, 2009.

A. phalloides (Fr.) Link - na ziemi; GF; oddz. 181b; IX 2005.

A. porphyria Alb. \& Schwein. - na torfie; VuPn; oddz. 182a; IX-X 2008-2009.

A. rubescens Pers. - na ziemi; GF, LF; oddz. 181b, 182b; VIII-X 2004-2006, 2007.

Armillaria solidipes Peck [ $=A$. ostoyae (Romagn.) Herink] - na pniach i pniakach; VuBe; oddz. 181a, c; X-XI 2007-2009.

Auricularia auricula-judae (Bull.) J. Schröt. - na kłodach Fagus; GF, LF; oddz. 181b, c, 182b; VI-IX 2005-2006.

Auriscalpium vulgare Gray - na szyszkach Pinus; VuPn; oddz. 182a; IV-VII, X 2008-2009.

Baeospora myosura (Fr.) Singer - na szyszkach Pinus; VuPn; oddz. 182a; X-XI 2008.

Bjerkandera adusta (Willd.) P. Karst. - na martwych pniakach i pniach; $G F, L F, V u B e$; oddz. 181b, c, 182b; IV-XI 2004-2009.

Boletus edulis Bull. - na ziemi; oddz. 181c; IX 2008.

B. erythropus Pers. - na ziemi; GF, LF; oddz. 181b, 182b; VIII-X 2004-2006; 2008-2009.

Calocera cornea (Batsch) Fr. - na martwych gałęziach i kłodach; VuBe; oddz. 181b, c; IX 20072009.

C. viscosa (Pers.) Fr. - na pniakach Pinus; VuPn; oddz. 182a; IX 2009.

Calvatia excipuliformis (Scop.) Perdeck - na ziemi; oddz. 181c; IX 2004.

Cantharellus cibarius Fr. - na ziemi; GF, LF; oddz. 181b, 182b; VII, IX 2004-2005, 2007.

Chlorophyllum rhacodes (Vittad.) Vellinga [=Macrolepiota rhacodes (Vittad.) Singer var. rhacodes] - na ziemi; oddz. 182b; IX 2006.

Chondrostereum purpureum (Pers.) Pouzar - na martwych pniakach i pniach Betula; VuBe, VuPn; oddz. 181b, c, 182a; VIII-XI 2004, 2008-2009.

Chroogomphus rutilus (Schaeff.) O.K. Mill. - na torfie i wśród mchów; VuPn; oddz. 182a; IX 2008-2009.

Clavulina coralloides (L.) J. Schröt. - na ziemi; oddz. 181b; X 2007.

Clitocybe clavipes (Pers.) P. Kumm. - wśród mchów; VuPn; oddz. 182a; IX-X 2007-2009.

C. nebularis (Batsch) P. Kumm. - na ziemi; LF; oddz. 182b; XI 2005.

C. vibecina (Fr.) Quél. - na ściółce; ErSp, VuPn; oddz. 181a, 182a; X-XI 2007-2009.

Collybia cirrata (Schumach.) Quél. - na starych owocnikach grzybów, wśród mchów i ściółki; ErSp; oddz. 181a; IX-X 2007-2009.

C. cookei (Bres.) J.D. Arnold - na starych owocnikach grzybów, wśród mchów i ściółki; ErSp, VuBe; oddz. 181a, c; VIII-X 2007-2009.

Coprinellus micaceus (Bull.) Vilgalys, Hopple \& Jacq. Johnson [=Coprinus micaceus (Bull.) Fr.] - na zmurszałej kłodzie; GF; oddz. 181b; VII-IX 2004-2005.

Coprinus comatus (O.F. Müll.) Pers. - na ziemi; 181c; VIII 2004, 2007.

Cortinarius acutus (Pers.) Fr. - wśród mchów pod Pinus; ErSp, VuPn; oddz. 181a, 182a; IX-X 2009. R.

C. armillatus (Fr.) Fr. - na torfie, pod Betula; VuBe; oddz. 181c; IX 2005.

C. cinnamomeoluteus P.D. Orton - na torfie; VuPn; oddz. 182a; IX 2009.

C. flexipes (Pers.) Fr. - na torfie, pod Pinus i Betula; ErSp, VuPn, VuBe; oddz. 181a, c, 182a; VIII-XI 2007-2009.

C. fulvescens Fr. - na torfie, wśród mchów, pod Pinus; ErSp, VuPn; oddz. 181a, 182a; IX-XI 2007-2009. E.

C. huronensis Ammirati \& A.H. Sm. - wśród Sphagnum; ErSp, LeSm, VuPn, VuBe; oddz. 181a, c, 182a; VII-XI 2007-2009.

C. rubellus Cooke [=C. speciosissimus Kühner \& Romagn.] - na torfie, wśród mchów; VuPn; oddz. 182a; VIII-IX 2009.

C. obtusus (Fr.) Fr. - na ściółce iglastej, wśród mchów, pod Pinus; ErSp; oddz. 181a; IX-X 20082009. 
C. semisanguineus (Fr.) Gillet - na torfie, wśród ściółki i mchów; VuPn; oddz. 182a; X 2005.

C. violaceus (L.) Gray - na ziemi, pod Betula i Populus tremula; w sąsiedztwie VuBe; oddz. 181c; IX 2008-2009. V.

Crepidotus variabilis (Pers.) P. Kumm. - na gałązkach; VuBe; oddz. 181b, c, 182b; VII-X 20042005, 2007-2009.

Crucibulum laeve (Huds.) Kambly - na drobnych gałązkach; oddz. 181c; IX 2004.

Cystoderma amianthinum (Scop.) Fayod - na torfie i wśród mchów; VuPn; oddz. 182a; IX-X 2007-2009.

Dacryomyces stillatus Nees - na martwych gałęziach, kłodach, pniakach i pniach; ErSp, GF, LF, VuBe, VuPn; oddz. 181a, b, c, 182a, b; VII-X 2004-2005, 2007-2009.

Daedaleopsis confragosa (Bolton) J. Schröt. - na martwych pniach Betula; VuBe; oddz. 181c; IV-XI 2007-2009.

Entoloma cetratum (Fr.) M.M. Moser - na torfie, wśród mchów; VuBe, VuPn; oddz. 181c, 182a; V, IX-XI 2007-2009.

E. conferendum (Britzelm.) Noordel. var. conferendum - wśród Sphagnum; VuPn; oddz. 182a; X 2008 .

E. sericatum (Britzelm.) Sacc. - na torfie i wśród mchów; ErSp, VuBe, VuPn; oddz. 181a, c, 182a; VIII-X 2007-2009.

Exidia plana Donk - na martwych gałązkach, gałęziach i kłodach; $G F, L F, V u B e$; oddz. 181b, c, 182b; IV-V, X-XI 2004-2005, 2008-2009.

Exobasidium karstenii Sacc. \& Trotter - na liściach i łodygach Andromeda polifolia; VuPn; oddz. 182a; VIII-IX 2008-2009.

E. vaccinii (Fuckel) Woronin - na liściach i łodygach Vaccinium vitis-idaea; VuPn; oddz. 182a; VIII-XI 2007-2009.

Fomes fomentarius (L.) Fr. - na gałęziach, kłodach i pniach Betula; GF, LF, VuPn, VuBe; oddz. 181a, b, c, 182a, b; IV-XI 2004-2009.

Fomitiporia punctata (P. Karst.) Murrill - na pniu Salix; oddz. 181c; VI-XI 2004-2005.

Fomitopsis pinicola (Sw.) P. Karst. - na kłodach i martwych pniach Pinus; VuPn; oddz. 182a, b; IV-XI 2007-2009.

Galerina calyptrata P.D. Orton - wśród mchów; EaSp, ErSp, VuPn; oddz. 181a, 182a; IX-X 2007-2009.

G. cinctula P.D. Orton - wśród Sphagnum i Erica tertralix; ErSp; oddz. 181a; IX 2008.

G. hypnorum (Schrank) Kühner - wśród mchów; VuPn; oddz. 182a; IX-XI 2007-2009.

G. jaapii A.H. Sm. \& Singer - wśród mchów; VuBe; oddz. 181c; X 2009. E.

G. paludosa (Fr.) Kühner - wśród Sphagnum; Cala, Caro, EaSp, ErSp, LeSm, Rhal, Spma, VuBe; oddz. 181a, c, 182a, b; V-X 2004-2009. R.

G. sphagnorum (Pers.) Kühner - wśród Sphagnum; Cala, EaSp, Spma; oddz. 181a, 182b; IX-X 2004-2005, 2008-2009. R.

G. tibiicystis (G.F. Atk.) Kühner - wśród Sphagnum; Cala, Caro, EaSp, ErSp, Rhal, Spma, VuBe; oddz. 181a, c, 182b; VI-X 2004-2009.

G. vittiformis (Fr.) Singer - wśród mchów; ErSp, VuBe, VuPn; oddz. 181a, c, 182a; IX-XI 20072009.

Ganoderma lipsiense (Batsch) G.F. Atk. - na kłodzie Fagus; GF, LF; oddz. 181b, 182b; IV-XI 2004-2009.

Gymnopilus hybridus (Bull.) Maire - na murszejącej kłodzie; VuPn; oddz. 182a; X 2007-2009.

Gymnopus androsaceus (L.) J.L. Mata \& R.H. Petersen - na opadłych igłach i drobnych gałązkach drzew iglastych oraz na Carex i Eriophorum; Cala, ErSp, LeSm, Spma, VuBe, VuPn; oddz. 181a, c, 182a, b; VII-XI 2004, 2007-2009.

G. confluens (Pers.) Antonín, Halling \& Noordel. - na ściółce; GF; oddz. 181b; IX 2005.

G. dryophilus (Bull.) Murrill - na ściółce; GF, $L F$, VuBe, VuPn; oddz. 181b, c, 182a, b; VI-XI 2004-2009. 
G. peronatus (Bolton) Gray - na ściółce iglastej i liściastej; $L F, V u B e, V u P n$; oddz. 181b, c, 182b, a; IX-X 2007-2009.

Hapalopilus nidulans (Fr.) P. Karst. - na gałęzi Quercus; oddz. 181b; VIII 2005.

Hebeloma cf. crustuliniforme (Bull.) Quél. s. lato - na torfie; VuPn; oddz. 182a; VIII-IX 20072008.

H. circinans Quél. Sacc. [ $=$ H. longicaudum (Pers.: Fr.) P. Kumm.] - na torfie i wśród Sphagnum; VuBe, VuPn; oddz. 181c, 182a; X 2007-2009.

H. cf. sacchariolens Quél. - na torfie; ErSp, VuBe; oddz. 181a, c; IX-X 2008.

Heterobasidion annosum (Fr.) Bref. - na pniaku Pinus; oddz. 182b; IV-XI 2004-2005.

Hohenbuehelia atrocoerulea (Fr.) Singer - na martwych gałęziach i kłodach Betula; VuBe; oddz. 181c; VIII-IX 2009.

Hydnum repandum L. - na ziemi; oddz. 182a; IX 2005.

Hygrophoropsis aurantiaca (Wulfen) Maire - na torfie; VuBe, VuPn; oddz. 181c, 182a, b; IX- XI 2007-2009.

Hymenochaete rubiginosa (Dicks.) Lév. - na drewnie Quercus; oddz. 182b; IX 2005.

Hymenochaetopsis tabacina (Sowerby) S.H. He \& Jiao Yang [=Hymenochaete tabacina (Sowerby) Lév.] - na gałązkach i gałęziach Salix; oddz. 181c; IV-X 2004-2005. R.

Hyphoderma setigerum (Fr.) Donk - na martwych gałęziach; oddz. 182b; IX 2004.

Hypholoma capnoides (Fr.) P. Kumm. - na pniakach Pinus; VuPn; oddz. 182a; IX-XI 2007-2009.

H. elongatum (Pers.) Ricken - wśród Sphagnum i innych mchów; Cala, Caro, EaSp, ErSp, LeSm, Rhal, Spma, VuBe, VuPn; oddz. 181a, c, 182a, b; IX-XI 2004-2006, 2007-2009. R.

H. fasciculare (Huds.) P. Kumm. - na pniakach i u podstawy pni drzew; $G F, L F$; oddz. 181b, c, 182b; VI-X 2004-2006.

H. lateritium (Schaeff.) P. Kumm. - na pniakach Betula; VuPn; oddz. 182a; V, XI 2008.

H. udum (Pers.) Quél - na torfie i wśród Sphagnum; Cala, Caro, EaSp, ErSp, LeSm, Rhal, Spma, VuBe, VuPn; oddz. 181a, c, 182a, b; VIII-XI 2004-2006, 2007-2009. R.

Imleria badia (Fr.) Vizzini [=Xerocomus badius (Fr.) E.-J. Gilbert] - na ziemi; GF, LF; oddz. 181b, 182b; IX-X 2004-2009.

Inocybe geophylla (Sowerby) P. Kumm. var. geophylla - na ziemi; oddz. 181c; IX 2004.

I. lacera (Fr.) P. Kumm. - na ziemi; oddz. 182a; IX 2005.

I. lanuginosa (Bull.) P. Kumm. - na torfie, pod Pinus; VuPn; oddz. 182a; IX-X 2008-2009.

Inonotus obliquus (Ach. ex Pers.) Pilát - na martwych i żywych pniach Betula; VuBe; oddz. 181c; IV-XI 2007-2009. R, Ch.

Kuehneromyces mutabilis (Schaeff.) Singer \& A.H. Sm. - na pniakach; oddz. 181b, c, 182b; VIII-X 2004-2006.

Laccaria amethystea (Bull.) Murrill - na ziemi; GF, LF; oddz. 181b, 182b; IX-X 2004-2005, 2008-2009.

L. lacccata (Scop.) Cooke - na ziemi; oddz. 182b; IX 2004.

L. proxima (Boud.) Pat. - na torfie i wśród Sphagnum; Cala, Caro, EaSp, ErSp, LeSm, Spma, VuPn, VuBe; oddz. 181a, c, 182a, b; V-XI 2004-2005, 2007-2009.

Lactarius blennius (Fr.) Fr. - na ziemi, pod Fagus; GF, LF; oddz. 181b, 182b; IX-X 2004-2005.

L. deliciosus (L.) Gray - wśród Sphagnum, pod Pinus; VuBe; oddz. 181 c; IX 2008.

L. glyciosmus (Fr.) Fr. - wśród Sphagnum, pod Betula; VuBe; oddz. 181c; IX-X 2007-2009.

L. helvus (Fr.) Fr. - na torfie i wśród Sphagnum, pod Pinus i Betula; Cala, Caro, EaSp, ErSp, LeSm, VuPn; oddz. 181a, c, 182a, b; VII-XI 2004-2005, 2007-2009.

L. lacunarum (Romagn.) J.E. Lange ex Hora - na torfie, pod Alnus i Betula; VuBe; oddz. 181c; IX 2008. E.

L. necator (Bull.) Pers. - na torfie, pod Betula; VuBe; oddz. 181c; IX 2007-2009.

L. pubescens Fr. - na ziemi, pod Betula; oddz. 181c; IX 2005.

L. quietus (Fr.) Fr. - na ziemi, pod Quercus; GF, LF; oddz. 181b, 182b; VIII-IX 2004-2005.

L. rufus (Scop.) Fr. - na torfie, pod Pinus; VuPn; oddz. 182a; X-XI 2008-2009. 
L. tabidus Fr. - na ziemi, torfie i wśród Sphagnum, pod Betula; Cala, EaSp, ErSp, Rhal, VuBe, VuPn; oddz. 181a, c, 182a, b; VII-X 2004, 2007-2009.

L. vietus (Fr.) Fr. - na torfie, pod Betula; oddz. 181c; IX 2004.

Leccinum aurantiacum (Bull.) Gray - na ziemi, pod Populus tremula; oddz. 181c; IX 2009.

L. niveum (Fr.) Rauschert - na torfie, wśród Sphagnum, pod Betula; Cala, ErSp, Rhal, VuBe, VuPn; oddz. 181a, c, 182a, b; VIII-X 2004-2005, 2007-2009. V.

L. scabrum (Bull.) Gray - na ziemi, pod Betula; oddz. 181b; VIII-XI 2007-2009.

L. versipelle (Fr. \& Hök) Snell - na ziemi, pod Betula; oddz. 181b; VIII-X 2007-2009.

Lentinellus cochleatus (Pers.) P. Karst. - na spróchniałym pniu Betula; VuBe; oddz. 181c; IX 2007-2008.

Lepista flaccida (Sowerby) Pat. - na ziemi; VuPn; oddz. 182a; IX-XI 2007-2009.

L. nuda (Bull.) Cooke - na ziemi; GF; oddz. 181b; X 2004.

Lichenomphalia umbellifera $(\mathrm{L}$.) Redhead, Lutzoni, Moncalvo \& Vilgalys [=Omphalina ericetorum (Pers.) Lange] - wśród mchów i porostów; VuBe, VuPn; oddz. 181c, 182a; X 2008-2009.

Lycoperdon perlatum Pers. - na ziemi; oddz. 181b, 182b; VIII-X 2008-2009.

L. pyriforme Schaeff. - na pniaku; oddz. 181b; VIII-XI 2009.

Macrolepiota procera (Scop.) Singer - na ziemi; oddz. 181c; IX 2004-2005.

Macrotyphula contorta (Holmsk.) Rauschert [=Clavariadelphus fistulosus (Holmsk.) Corner] na martwych gałązkach Betula; oddz. 181b; IX-X 2005. R.

Megacollybia platyphylla (Pers.) Kotl. \& Pouzar - na zmurszałej kłodzie Betula; GF, LF, VuBe; oddz. 181b, c, 182b; VIII-IX 2004-2008.

Mycena acicula (Schaeff.) P. Kumm. - na ziemi, wśród mchów; IX 2004.

M. cinerella (P. Karst.) P. Karst. - na ściółce i wśród mchów; VuPn; oddz. 182a; X-XI 2007-2009.

M. epipterygia (Scop.) Gray var. epipterygia - na ściółce, wśród mchów; ErSp, LeSm, VuBe, VuPn; oddz. 181a, c, 182a; IX-XI 2007-2009.

M. filopes (Bull.) P. Kumm. - na ściółce; VuPn; oddz. 182a; IX, XI 2008-2009.

M. galericulata (Scop.) Gray - na pniakach, gałęziach i kłodach; ErSp, GF, LF, VuBe, VuPn; oddz. 181a, b, c, 182a, b; VIII-XI 2004-2009.

M. galopus (Pers.) P. Kumm. - na ściółce, wśród Sphagnum; Cala, Caro, ErSp, LeSm, Spma, VuBe, VuPn; oddz. 181a, c, 182a, b; V-XI 2004-2009.

M. haematopus (Pers.) P. Kumm. var. haematopus - na murszejących kłodach; GF, $L F$, VuBe; oddz. 181b, c, 182b; IX-X 2004-2006, 2009.

M. megaspora Kauffman \& A.H. Sm. - wśród Sphagnum; VuBe, VuPn; oddz. 181c, 182a; IX-X 2008-2009. V.

M. pura (Pers.) P. Kumm. - na ściółce; GF, LF; oddz. 181b, 182b; VIII-X 2004-2005.

M. sanguinolenta (Alb. \& Schwein.: Fr.) P. Kumm. - na torfie i ściółce; ErSp, LeSm, VuBe, VuPn; oddz. 181a, c, 182a; VIII-X 2007-2009.

M. stipata Maas Geest. \& Schwöbel - na murszejących kłodach i pniakach Pinus; VuBe, VuPn; oddz. 181c, 182a; IV-V, X-XI 2007-2009.

M. vitilis (Fr.) Quél. - na martwych, drobnych gałązkach; GF, ErSp, VuPn; oddz. 181a, b, 182a; VIII-XI 2004, 2008-2009.

M. vulgaris (Pers.) P. Kumm. - na torfie i ściółce; ErSp, VuPn; oddz. 181a, 182a; X-XI 2008-2009.

M. zephirus (Fr.) P. Kumm. - na torfie i ściółce; $G F, L F, V u B e, V u P n$; oddz. 181b, c, 182a, b; X-XI 2004-2005, 2007-2009.

Omphalina gerardiana (Peck) Singer - wśród Sphagnum; Cala, Caro, EaSp, Rhal, Spma; oddz. 181a, 182a, b; VI-X 2004-2005, 2007-2009. V.

Oudemansiella mucida (Schrad.) Höhn. - na kłodach Fagus; GF, LF; oddz. 181b, 182b; IX 2004-2005.

Panellus mitis (Pers.) Singer - na martwych gałęziach Pinus; oddz. 182a, b; X 2005.

P. stipticus (Bull.) P. Karst. - na pniaku Quercus; oddz. 82b; IX-X 2004. 
Paxillus involutus (Batsch) Fr. - na torfie, pod Betula i Pinus; ErSp, GF, LF, VuPn; oddz. 181a, b, 182a, b; IX-XI 2004-2009.

Peniophora cinerea (Pers.) Cooke - na martwych gałązkach Betula; oddz. 181b, c, 182b; VI-XI 2004-2006, 2009.

P. quercina (Pers.) Cooke - na gałęzi Quercus; oddz. 181b, 182b; VII-X 2004.

Phaeomarasmius erinaceus (Fr.) Scherff. ex Romagn. - na martwych gałązkach Salix; oddz. 181c; VIII 2007. R.

Phallus impudicus L. - na ziemi; GF; oddz. 181b; VIII-IX 2004-2005.

Phanerochaete sanguinea (Fr.) Pouzar - na martwej gałązce; oddz. 182a; X 2004.

Phellinus ferruginosus (Schrad.) Pat. - na gałęzi Quercus; LF; oddz. 182b; IX 2004.

Phlebia radiata Fr. - na pniakach i kłodach Betula i Fagus; $L F$, VuBe; oddz. 181b, c, 182b; XI 2005, 2008-2009.

Ph. tremellosa (Schrad.) Nakasone \& Burds. - na murszejących kłodach Betula; VuBe; oddz. 181c; VIII-X 2007.

Pholiota flammans (Batsch) P. Kumm. - na kłodzie Pinus; oddz. 182a; IX 2004.

Piptoporus betulinus (Bull.) P. Karst. - na pniach i kłodach Betula; VuBe, VuPn; oddz. 181b, c, 182a, b; IV-XI 2004-2009.

Pluteus cervinus (Schaeff.) P. Kumm. - na pniakach i kłodach Betula; VuBe, VuPn; oddz. 181c, 182a; IX-X 2007-2009.

Polyporus ciliatus Fr. - na gałązkach i gałęziach Betula; VuBe; oddz. 181c; IV-VI 2008-2009.

P. varius (Pers.) Fr. - na gałęzi Fagus; GF, LF; oddz. 181b, 182b; VIII-X 2004-2005.

Postia caesia (Schrad.) P. Karst. - na martwych i murszejących gałęziach i kłodach Pinus; VuPn; oddz. 182a; VII-XI 2008-2009.

Psathyrella candolleana (Fr.) Maire - na ziemi; oddz. 181c; IX 2005.

P. piluliformis (Bull.) P.D. Orton - na pniaku; oddz. 181b; IX 2005.

Pseudoboletus parasiticus (Bull.) Šutara [=Xerocomus parasiticus (Bull.) Quél.] - na owocnikach Scleroderma citrinum; VuBe; oddz. 181a; IX-X 2009. R, Ch.

Pseudomerulius aureus (Fr.) Jülich - na kłodzie Pinus; oddz. 182a; IX 2005. R.

Ramaria stricta (Pers.) Quél. - na ziemi; GF, LF; oddz. 181b, 182b; IX-X 2004-2006, 2008.

Rhodocollybia butyracea (Bull.) Lennox f. butyracea - na torfie i wśród mchów; VuBe, VuPn; oddz. 181b, c, 182a; X-XI 2008-2009.

R. maculata (Alb. \& Schwein.) Singer - na torfie; ErSp, VuPn; oddz. 181a, 182a; IX-X 2004$2005,2009$.

Rickenella fibula (Bull.) Raithelh. - na mchach; VuBe, VuPn; oddz. 181c, 182a; VI-X 2007-2009.

Roridomyces roridus (Fr.) Rexer [=Mycena rorida (Fr.) Quél.] - na ściółce, wśród mchów; oddz. 182a; IX-X 2004-2005.

Russula aeruginea Lindblad - na ziemi, pod Betula; oddz. 181b, c; IX-X 2004-2005.

R. betularum Hora - na torfie, pod Betula; ErSp, VuBe, VuPn; oddz. 181a, c, 182a; IX-X 20072009.

R. claroflava Grove - na torfie, pod Betula; ErSp, VuBe, VuPn; oddz. 181a, c, 182a; VIII-XI 2007-2009.

R. cyanoxantha (Schaeff.) Fr. - na ziemi; oddz. 181b; IX 2005.

R. decolorans (Fr.) Fr. - na torfie, pod Pinus; VuPn; oddz. 182a; IX 2005, 2009.

R. emetica (Schaeff.) Pers. - na torfie i wśród Sphagnum, pod Pinus; ErSp, VuPn; oddz. 181a, 182a; IX-XI 2007-2009.

R. fragilis Fr. - na ziemi; oddz. 181b; IX-X 2005.

R. nigricans Fr. - na ziemi; GF, LF; oddz. 181b, 182b; VIII-XI 2004-2005, 2008-2009.

R. nitida (Pers.) Fr. - wśród Sphagnum; VuBe; oddz. 181c; VI, IX-X 2008-2009.

R. ochroleuca Pers. - na ziemi; oddz. 182b; IX 2005.

R. paludosa Britzelm. - na torfie, pod Pinus; ErSp, VuPn; oddz. 181a, 182a; IX 2008. 
R. xerampelina (Schaeff.) Fr. - na torfie, pod Pinus; ErSp, VuPn; oddz. 181a, 182a; VIII-X 20082009.

Schizophyllum commune Fr. - na pniakach i gałęziach; oddz. 181c; VII-X 2005, 2008.

Schizopora paradoxa (Schrad.) Donk - na zmurszałym drewnie; VuBe, VuPn; oddz. 181b, c, 182a, b; VII-XI 2008-2009.

Scleroderma citrinum Pers. - na torfie, wśród mchów, często wśród Sphagnum; VuBe; VuPn; oddz. 181c, 182a; XI-XI 2007, 2009.

Sphaerobolus stellatus Tode - na murszejących gałęziach i kawałkach drewna; VuBe, VuPn; oddz. 181c, 182a; IX-XI 2009.

Sphagnurus paluster (Peck) Redhead \& V. Hofst. [=Lyophyllum palustre (Peck) Singer, Tephrocybe palustris (Peck) Donk] - na Sphagnum; Cala, Caro, EaSp, LeSm, Rhal, Spma, VuBe; oddz. 181a, c, 182b; V-XI 2004-2009. V.

Stereum hirsutum (Willd.) Gray - na opadłych i murszejących gałęziach Betula; VuBe; oddz. 181b, c, 182b; VII-XI 2004-2009.

S. rugosum Pers. - na pniakach Betula i pniach Corylus; VuBe; oddz. 181b, c, 182b; IV-XI 20042009.

S. sanguinolentum (Alb. \& Schwein.) Fr. - na gałęziach Pinus; VuPn; oddz. 181b, 182a, b; IX-XI 2004-2009.

S. subtomentosum Pouzar - na gałęziach Salix; VuBe; oddz. 181c; IV-XI 2007-2009.

Strobilurus tenacellus (Pers.) Singer - na szyszkach Pinus; VuPn; oddz. 182a; IV-VI 2007-2009.

S. stephanocystis (Kühner \& Romagn. ex Hora) Singer - na szyszkach Pinus; VuPn; oddz. 182a; IV, VI 2008-2009.

Stropharia aeruginosa (Curtis) Quél. - na torfie; VuPn; oddz. 182a; IX 2009.

Suillus bovinus (L.) Roussel - na torfie, pod Pinus; ErSp, VuPn; oddz. 181a, 182a; IX-XI 20042005, 2007-2009.

S. flavidus (Fr.) Singer - na torfie, pod Pinus; ErSp, VuPn; oddz. 181a, 182a; IX-X 2007-2009. E, Ch.

S. variegatus (Sw.) Kuntze - na torfie, pod Pinus; ErSp, LeSm, VuPn; oddz. 181a, 182a; IX-XI 2007-2009.

Tapinella panuoides (Fr.) E.-J. Gilbert [=Paxillus panuoides (Fr.) Fr.] - na kłodzie Pinus; oddz. 182b; X 2004.

Thelephora terrestris Ehrh. - na torfie, wśród Sphagnum, na łodygach Carex i Eriophorum; EaSp, ErSp, LeSm, Spma, VuPn; oddz. 181a, 182a; IV-XI 2004, 2007-2009.

Trametes hirsuta (Wulfen) Pilát - na martwych gałęziach Betula; ErSp, VuBe, VuPn; oddz. 181a, c, 182a; IX-XI 2007-2009.

T. versicolor (L.) Lloyd - na pniakach i gałęziach Betula; oddz. 181b; IV-XI 2004-2005.

Tremella encephala Pers. - na owocnikach Stereum sanguinolentum i gałęziach Pinus; oddz. 182a; IX-X 2005.

T. mesenterica (Schaeff.) Retz. - na martwej gałęzi Quercus; oddz. 181b; IX 2005.

Trichaptum fuscoviolaceum (Ehrenb.) Ryvarden - na pniakach i kłodach Pinus; VuPn; oddz. 182a; IV-XI 2007-2009.

Tricholomopsis rutilans (Schaeff.) Singer - na pniaku Pinus; VuPn; oddz. 182b; IX 2005.

Tylopilus felleus (Bull.) P. Karst - na torfie, pod Pinus; VuPn; oddz. 182a; IX-X 2007-2009.

Vuilleminia comedens (Nees) Mraire - na gałęzi Quercus; GF, LF; oddz. 181b, 182b; IV-XI 2004-2009.

Xerocomellus chrysenteron (Bull.) Šutara - na ziemi; GF, LF; oddz. 181b, 182b; VIII-X 20042009.

Xerocomus subtomentosus (L.) Quél. - na ziemi; oddz. 182a; IX 2004.

Xeromphalina cauticinalis (Fr.) Kühner \& Maire - na ściółce iglastej; VuPn; oddz. 182a; IX 2004.

Xerula radicata (Relhan) Dörfelt - na korzeniach Fagus; oddz. 182b; IX 2004. 


\section{PODSUMOWANIE}

Na terenie rezerwatu „Torfowisko Toporzyk” stwierdzono łącznie 216 taksonów grzybów, w tym 20 znajdujących się na czerwonej liście grzybów wielkoowocnikowych w Polsce. Zarówno liczba odnotowanych gatunków, świadcząca o znacznej różnorodności macromycetes rezerwatu, jak i udział taksonów zagrożonych i chronionych, wskazują na dużą wartość przyrodniczą tego obiektu nie tylko pod względem florystycznym. Wiele $\mathrm{z}$ odnotowanych $\mathrm{w}$ rezerwacie gatunków grzybów charakteryzuje się dość szeroką skalą ekologiczną. Wyodrębnia się jednak grupa taksonów wykazująca wyraźny związek ze zbiorowiskami torfowiskowymi, zwłaszcza mszarnymi. Do tej grupy należą m.in.: Galerina paludosa, G. sphagnorum, G. tibiicystis, Hypholoma elongatum, H. udum i Sphagnurus paluster, rosnące wśród torfowców. Udział tych grzybów może świadczyć o pewnej trwałości odnawiających się zbiorowisk torfotwórczych.

\section{LITERATURA}

Brzeg A., Król S., Kuświk H., Melosik I., Urbański P. (1992): Dokumentacja naukowo-techniczna projektowanego rezerwatu przyrody „Torfowisko Toporzyk”. Wojewódzki Konserwator Przyrody. Koszalin (mskr.).

Brzeg A., Kuświk H., Melosik I., Urbański P. (1995): Flora i roślinność projektowanego rezerwatu przyrody „Torfowisko Toporzyk” w Drawskim Parku Krajobrazowym. Bad. Fizjogr. nad Polską Zach., B, 44: 51-76.

Herbichowa M. (2004): Torfowiska przejściowe i trzęsawiska (przeważnie z roślinnością z Scheuchzerio-Caricetea nigrae). [W:] Herbich J. (red.). Wody słodkie i torfowiska. Poradnik ochrony siedlisk i gatunków Natura 2000 - podręcznik metodyczny. T. 2: 147-157. Ministerstwo Środowiska. Warszawa.

Index Fungorum. http://www.indexfungorum.org (dostęp: 15.02.2017).

Jasnowska J., Jasnowski M. (1983): Pojezierze Zachodniopomorskie. Ss. 257. Ser. Przyr. Pol., Wiedza Powszechna. Warszawa.

Jasnowska J., Kowalski W.A., Zyska W. (2003-2004): Plan ochrony rezerwatu „Toporzyk”. Biur. Konserw. Przyr. w Szczecinie, Woj. Zachodniopomor., Nadl. Połczyn Zdrój. Szczecin (mskr.).

Kaźmierczakowa R., Bloch-Orłowska J., Celka Z., Cwener A., Dajdok Z., Michalska-Hejduk D., Pawlikowski P., Szczęśniak E., Ziarnek K. (2016): Polska czerwona lista paprotników i roślin kwiatowych. Ss. 44. Inst. Ochr. Przyr. PAN. Kraków.

Koźmiński C., Michalska B., Czarnecka M. (2007): Klimat województwa zachodniopomorskiego. Ss. 147. AR Szczecin, Uniw. Szczeciński. Szczecin.

Markowski R., Buliński M. (2004): Ginące i zagrożone rośliny naczyniowe Pomorza Gdańskiego. Acta Bot. Cassub., Monogr., 1: 1-75.

Matuszkiewicz W. (2001): Przewodnik do oznaczania zbiorowisk roślinnych Polski. Ss. 537. Ser. Vademecum Geobot., 3. Wyd. Nauk. PWN. Warszawa.

Mirek Z., Piękoś-Mirkowa H., Zając A., Zając M. (2002): Flowering Plants and Pteridophytes of Poland. A checklist. Ss. 442. Ser. Biodiversity of Poland, 1. W. Szafer Inst. of Bot., Pol. Acad. of Sci. Kraków.

Ochyra R., Żarnowiec J., Bednarek-Ochyra H. (2003): Census Catalogue of Polish Mosses. Ss. 372. Ser. Biodiversity of Poland, 3. W. Szafer Inst. of Bot., Pol. Acad. of Sci. Kraków. 
Robert V., Stegehuis G., Stalpers J. (2005): The MycoBank engine and related databases. http:// www.mycobank.org (dostęp: 15.02.2017).

Rozporządzenie Ministra Środowiska z dnia 9 października 2014 r. w sprawie ochrony gatunkowej grzybów. Dz.U. z dnia 16 października 2014 r., poz. 1408.

Sotek Z. (2010): Distribution patterns, history, and dynamics of peatland vascular plants in Pomerania (NW Poland). Biodiv. Res. Conserv., 18: 1-82.

Stasińska M. (2011): Macrofungi of raised and transitional bogs of Pomerania. Monogr. Bot., 101: $1-142$.

Stasińska M., Sotek Z. (2010): Zróżnicowanie macromycetes mszaru wrzoścowego Erico-Sphagnetum medii (Schwick 1933) Moore 1968 - wstępne wyniki badań. IMUZ. Woda - Środowisko - Obszary Wiejskie, 10, 3(31): 271-282.

Wojewoda W., Ławrynowicz M. (2006): Red list of the macrofungi in Poland. - Czerwona lista grzybów wielkoowocnikowych w Polsce. [W:] Mirek Z., Zarzycki K., Wojewoda W., Szeląg Z. (red.). Red list of plants and fungi in Poland. - Czerwona lista roślin i grzybów Polski: 53-70. W. Szafer Inst. of Bot., Pol. Acad. of Sci. Kraków.

Zabawski J., Matuła J. (1975): Interesujące torfowisko z wrzoścem bagiennym Erica tetralix w okolicach Połczyna Zdroju. Chrońmy Przyr. Ojcz., 31(2): 31-34.

Zając M., Zając A. (2006): Western element in the vascular flora of Poland. Biodiv. Res. Conserv., 1-2: 57-63.

Zarządzenie Ministra Ochrony Środowiska, Zasobów Naturalnych i Leśnictwa z dnia 12 listopada 1996 r. Monitor Polski nr 75, poz. 692 z dnia 9 grudnia 1996 r.

Żukowski W., Jackowiak B. (1995): Lista roślin naczyniowych ginących i zagrożonych na Pomorzu Zachodnim i w Wielkopolsce. [W:] Żukowski W., Jackowiak B. (red.). Ginące i zagrożone rośliny naczyniowe Pomorza Zachodniego i Wielkopolski: 9-96. Pr. Zakł. Taksonomii Roślin UAM, 3. Bogucki Wyd. Nauk. Poznań.

\section{MACROFUNGI OF THE “TORFOWISKO TOPORZYK” NATURE RESERVE (NW POLAND)}

\section{Sum mary}

In the years 2004-2009, mycological studies were conducted in the "Torfowisko Toporzyk" reserve, located in the Połczyn-Zdrój commune. The purpose of the studies was to determine the species occurrence and diversity of macroscopic fungi within the reserve. For observation, permanent plots were designated in patches of plant associations: Caricetum lasiocarpae, Eriophoro angustifolii-Sphagnetum recurvi, Erico-Sphagnetum medii, Rhynchosporetum albae, Vaccinio uliginosi-Betuletum pubescentis and Vaccinio uliginosi-Pinetum. In the case of the communities: Caricetum rostratae, Ledo-Sphagnetum magellanici, Sphagnetum magellanici, Galio odorati-Fagetum and Luzulo pilosae-Fagetum, the route method was used. As a result of the studies, 216 fungal species were identified, 17 Ascomycota and 199 Basidiomycota. Twenty of the recorded species are included on the Polish red list of macrofungi (Wojewoda, Lawrynowicz 2006), according to their threat categories. Four species: Cortinarius fulvescens, Galerina jaapii, Lactarius lacunarum and Suillus flavidus belong to 
the category E (endangered); five species, e.g. Cortinarius violaceus, Leccinum niveum, Mycena megaspora to V (vulnerable), and eleven to R (rare), e.g. Galerina sphagnorum, Phaeomarasmius erinaceus and Pseudomerulius aureus. Three of the threatened species are under partial protection: Inonotus obliquus, Pseudoboletus parasiticus and Suillus flavidus. Among the plant associations, Vaccinio uliginosi-Pinetum (87) and Vaccinio uliginosi-Betuletum pubescentis (77), turned out to be the richest in fungi, whereas the fewest taxa were found in Rhynchosporetum albae (8).

Both the number of identified species, indicating a considerable diversity of macromycetes within the reserve, and the share of threatened and protected taxa, point to the great natural value of this reserve, not only with regard to its floristic composition. 